\title{
Molecular Simulation of Enantiodifferentiating Photoisomerization of Cyclooctene by Chiral Sensitizers
}

\author{
Hiromitsu HASHIMOTO, Tetsuro SHIMO*, Mikito ATSUCHI, Masaru MITSUSHIO \\ and Kenichi SOMEKAWA*
}
Department of Applied Chemistry and Chemical Engineering, Faculty of Engineering, Kagoshima University 1-21-40 Korimoto, Kagoshima 890-0065, Japan
*e-mail: shimo@apc.kagoshima-u.ac.jp,some@apc.kagoshima-u.ac.jp

(Received: June 9, 2008; Accepted for publication: September 16, 2008; Advance publication: October 24, 2008)

For theoretical study on interesting enantiodifferenciating photoisomerization of $(Z)$-cyclooctene $(\mathbf{1 Z})$ to $(E)$-cyclooctene $(\mathbf{1 E})$ by chiral benzenepolycarboxylates $(\mathbf{2 a})$ in solution, the profiles of energy and stereochemical change of the sensitized photoisomerization were inferred by molecular simulation by use of MOPAC-PM5 method.

The energy, stereochemical change and equilibrium of the ground states and excited singlet states of $\mathbf{1 Z}$, $\mathbf{1 E}$, tetramethyl 1,2,4,5-benzenetetracarboxylate (2b), dimethyl 3,5-bis(trifluoromethyl)benzoate (2c), and $(R)$ - and $(S)$-dimenthyl 3,5-bis(trifluoromethyl)benzoate (2dr and $\mathbf{2 d s}$ ) as a chiral model of $\mathbf{2 a}$ were first interpreted. $\mathbf{1 Z}$ has an asymmetric conformer and the isomerization to $(R)$ - or $(S)$-1E may be dependent on preferential one-side rotation. Inferred exciplexes $(\mathbf{E x 1})$ such as $\mathbf{1 Z} \cdot \mathbf{2} \mathbf{b}^{*}\left(\mathbf{2} \mathbf{b}^{*}\right.$ : excited singlet state of $\left.\mathbf{2 b}\right)$ occur via energy transfers through some interactions to bring the next loose exciplexes $(\mathbf{E x 2})$ such as $\mathbf{1 E}^{*} \cdot \mathbf{2} \mathbf{b}$, which give $\mathbf{1 E}$ at the respective 1E/1Z ratio. The Ex1 and Ex2 structures were inferred to possess proper $\pi^{*} / \pi$ and $\mathrm{C}-\mathrm{H} / \pi$ interactions, ester $\mathrm{C}=\mathrm{O} / \mathrm{HC}$ (olefin and allyl) and $\mathrm{F} / \mathrm{H}$ hydrogen bondings, placed in parallel or alongside depending on the substituents. The photoisomerization may depend on the energy difference between the two exciplexes, and the transition state (TS) energy. The carbonyl groups and $\pi$ components on the enantiomeric polycarboxylates make selective hydrogen bondings, $\pi^{*} / \pi$ and $\mathrm{CH} / \pi$ interactions with the olefin and allyl groups on asymmetric 1Z, and bring diastereomeric Ex1. Those interaction accumulations are inferred to bring enantiodifferentiating energy transfer by one-side rotation (asymmetric environment) of the two molecules for $(R)-\mathbf{1 E}$ or $(S)-\mathbf{1 E}$ like enzymes.

Keywords: Molecular simulation, PM5, Enantiodifferentiation, Photoisomerization, Cyclooctene, Chiral sensitizer, Benzenepolycarboxylate

\section{Introduction}

\subsection{Chirality control}

The origin of biomolecular homochirality in the biosphere is one of the most interesting and contentious issues in the chemical evolution. The founders of stereochemistry, van't Hoff and Le Bel, had suggested the use of circularly polarized light for "absolute asymmetric synthesis (AAS)" in the 19th century. Many kinds of AAS for chiral control and for useful products were developed by use of peculiar chiral catalysts by several Nobel prizers, etc. in the last half of 20th century. The field of "asymmetric photochemistry" has however undergone an accelerated development only in the past 20 years. "Chiral Photochemistry" edited by Y. Inoue and
V. Ramamurthy [1] provides a range of articles with various aspects of controlling the chirality of photochemical reactions so-called "photochirogenesis". It contains, (1) direct asymmetric photochemistry with circularly polarized light,

(2) magnetochiral anisotropy in asymmetric photochemistry,

(3) enantiodifferentiating photosensitized reactions,

(4) chirality in photochromism,

(5) chiral photochemistry with transition metal complexes, and

(6) enantioselective photochemical reactions in various molecular aggregates or in the solid media, etc..

Very few authors however described the chiral mechanisms by the molecular orbital (MO) method. 


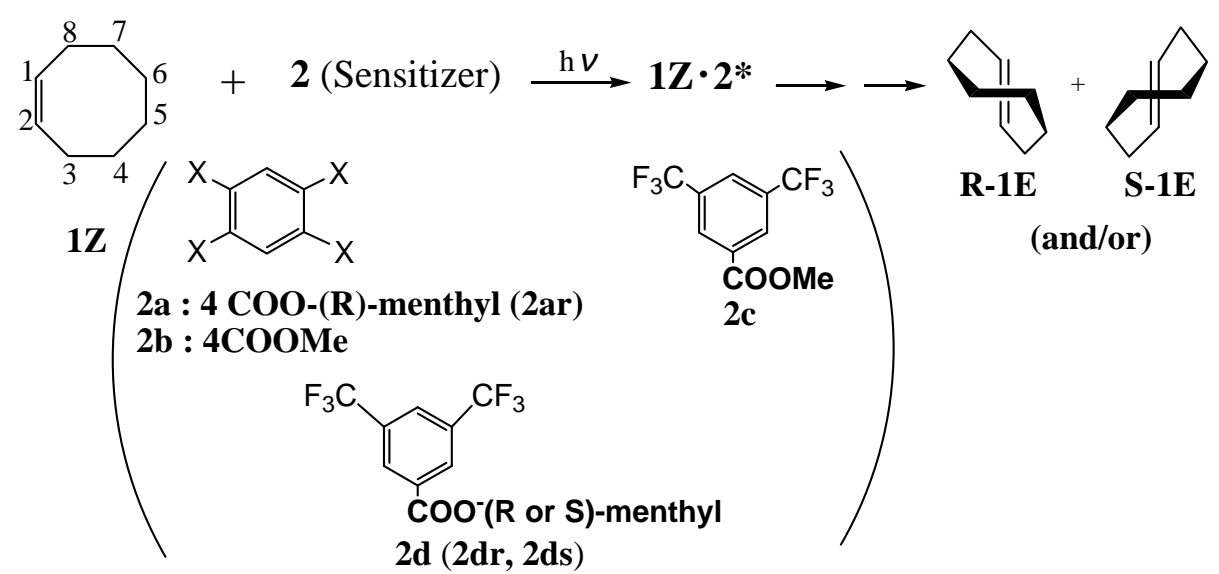

Scheme 1

\subsection{Enantiodifferentiating photoisomeriza- tion}

Various and interesting experimental studies by Inoue et al. $[1,2]$ on the enantiodifferentiating photosensitized reactions in solution, have stimulated our requisition for the mechanism and factors that control the stereochemical outcome of the enantio- and diastereodifferentiating process in the excited state. Namely, the sensitized enantiodifferentiating photoisomerizations (action by $\mathbf{2 a}$ in Scheme 1) by Inoue et al. [2-5] were especially appearing as a method of efficient chirality transfer. The chirality transfer is unusually effected by weak energies of the reaction temperature and pressure etc.. Development of "Entropy-control" reactions by the enantiodifferentiating photoisomerization is now in one of international big projects of ICORP. The biological application example of enantiomeric selectivity in the bringing of $(R)$-or $(S)$ - $E$-cyclooctene (1E) to ethylene (hormone) receptor antagonist is shown in section 3.4.

The conformation and energy profile at the stereodifferentiating processes in the excited state have however not been promoted. Dynamic molecular orbital (MO) method may be effective for understanding of the whole process [6, 7]. We have elucidated major factor and origin for peri-, site-, regio- and stereoselectivities of many kinds of photocycloadditions by frontier MO (FMO) [8] and transition state (TS) analysis using improved MOPAC-PM5 and UCIS (or UB3LYP) level of calculations [9-12]. The TS energy change by frontier orbital interactions, ionic interactions and hydrogen-bonding interactions is effective for the product-selectivities. PM5 is thought to be effective for calculation of such singlet excited photoreactions. We now elucidate the energy and stereochemical profile of photosensitized enantiodifferentiating $Z-E$ isomerization of cyclooctene by some benzenepolycarboxylates (2b-2d in Scheme 1) by molecular simulation using the MOPACPM5 level.

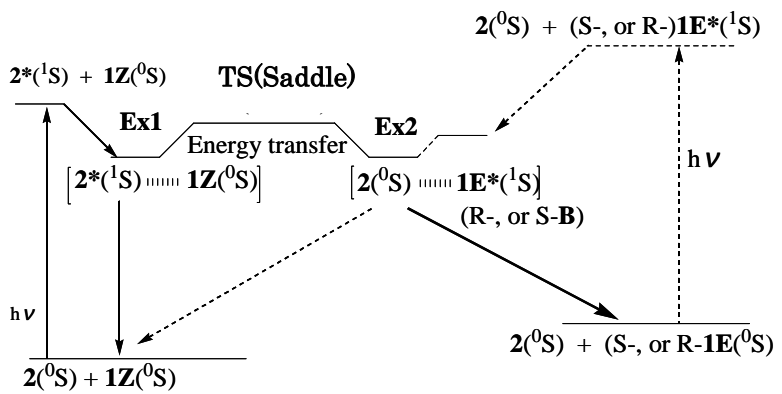

Figure 1. Process of sensitized enantiodifferentiating photoisomerization

The photosensitized isomerization process is supposed as the solid line via 2 exciplexes, Ex1 and Ex2 in Figure 1.

\section{Experimental data and calcula- tion method}

\subsection{Photoisomerization data}

Sensitized photoisomerization data of $\mathbf{1 Z}$ to $\mathbf{1 E}$ by many kinds of benzenecarboxylates and cyanobenzenes are given in the literature [3]. $\mathbf{1 E} / \mathbf{1 Z}$ ratio by $\mathbf{2 a}, \mathbf{2 b}, \mathbf{2 c}$ and 2d are nearly $0.01,0.1,0.6$, and 0.3 , respectively. The enantioselectivity, $(S)-\mathbf{1 E} /(R)-\mathbf{1 E}$ ratio by $\mathbf{2 a}$ is higher than the one by $\mathbf{2 d}$.

\subsection{Calculation}

All geometry optimization and energy minimum path at the interactions of the two substrates at ground states and excited singlet states were performed using PM5, 
which are available in the molecular orbital package WinMOPAC3.5 by Fujitsu Ltd. [13, 14].

(1) Keyword for PM5

Ground state: EF PM5 PRECISE, Excited singlet state: EF PM5 EXCITED SINGLET OPEN(2,2) [14], Saddle point: SADDLE PM5, Transition state: TS PM5, Normal mode analysis: FORCE ISOTOPE PRECISE (XYZ) [15].

(2) Two molecular interaction

Interactions between excited singlet state $2\left(\mathbf{2}^{*}\right)$ and cyclooctene $\mathbf{1}$ were calculated by approach of the proper parts of the two molecules [14]. The stable structures Ex1 in Figure 3, and Ex2 in Figure 5 etc., were optimized. The TS structures such as TS $(\mathbf{C})$ in Figure 3, C in Figure 5, Saddle in Figure 6, and TS in Figure 8 were checked by normal mode analysis with only imaginary frequency, which is evidence of the TS point. The frequency of the $\mathbf{C}$ point in Figure 4 was only imaginary: $-1125 \mathrm{~cm}^{-1}($ GNorm $=5.3)$. We need precise checking for other data (GNorm = 16-95) of "too large GNorm". They however seem to be reasonable similarly to Figure 5 . We utilize them for discussion.

The excited state MO of 2a was not obtained. It might be caused by the many alkyl conformations.

(3) Reasons of MOPAC-PM5 utilization and the accuracy MOPAC program has been improved in accuracy from PM3 to PM5 and PM6 [16], and has also advantages in cost-performance and consecutive graphs. The accuracy of PM5 for Diels-Alder reaction TS analysis is very good as well as one of B3LYP(/6-31+G(d)) [17, 18]. PM5 method was the best among semi-empirical ones and the usage could reproduce the crystal structure as well as B3LYP/LANL2DZ [19]. For TS analysis of photocycloaddition selectivities, PM5 method was also effective as well as UCIS (at singlet reactions) and UB3LYP (at triplet reactions) [10-12, 14].

\section{Results and discussion}

\subsection{Energy (HOF) diagram and stere- ochemical profile of $(Z)$ - and $(E)$ - cyclooctenes, $1 \mathrm{Z}$ and $1 \mathrm{E}$}

The heat of formation (HOF) of the lowest energy conformer of $\mathbf{1 Z}$ by PM5 method is $-14.7 \mathrm{kcal} / \mathrm{mol}$, and is $11.7 \mathrm{kcal} / \mathrm{mol}$ lower [3] than that of $\mathbf{1 E}$ (dihedral angle: $\Phi_{8123}=143^{\circ}$ ). Their relative results and stereochemistry are shown in Figure 2.

$\mathbf{1 Z}\left(\Phi_{8123}=-1.5^{\circ}, \Phi_{4567}=-107^{\circ}\right)$ has the asymmetry conformer $1 Z \mathbf{c}\left(\Phi_{8123}=1.5^{\circ}, \Phi_{4567}=107^{\circ}\right)$, and the conformation barrier $(4.0 \mathrm{kcal} / \mathrm{mol})$ for equilibrium may be concerned with the chiral sensitization by chiral sensitizers as shown in section 3.4. $\mathbf{1 Z}$ is correlated to $(R)-(-)-\mathbf{1 E}$ $\left(\Phi_{8123}=-143^{\circ}, \Phi_{4567}=103^{\circ}\right)$ by a quarter rotation.
Calculated TS point (relative $\mathrm{HOF}=61 \mathrm{kcal} / \mathrm{mol}$ ) between $\mathbf{1 Z}$ and $1 \mathbf{E}$ in Figure 2 was introduced by use of keyword SADDLE, and then TS. We have not succeeded in "normal mode with only imaginary frequency" which was shown at section 2.2.

The two excited singlet states $\left(\mathbf{1 Z}^{*}\right.$ and $\mathbf{1 E}^{*}(R$ or $S)$ ) of $\mathbf{1 Z}$ and $\mathbf{1 E}$ are intersected at $\Phi_{8123}=94^{\circ}$, and have other conformers. They explain the population data $(\mathbf{1 Z} / \mathbf{1 E} \fallingdotseq 0.5)$ at the direct photoisomerizations by Inoue et al. [3].

\subsection{Sensitized photoisomerization of $1 \mathrm{Z}$ to $1 E$ by $2 b^{*}$}

\subsubsection{Exciplex Ex1 formation between $1 Z$ and $2 b^{*}$}

Equation 1 and Figure 3 show the calculated energy diagram

$$
(\mathbf{1 Z}+\mathbf{2 b} \stackrel{h v}{\longrightarrow}) \mathbf{1 Z}+\mathbf{2} \mathbf{b}^{*} \rightarrow \mathbf{1 Z} \cdot \mathbf{2} \mathbf{b}^{*}(\mathrm{Ex} \mathbf{1})
$$

and stereochemical changes by approach between $\mathrm{C} 1$ $\left(\mathbf{2} \mathbf{b}^{*}\right)$ and $\mathrm{C} 1(\mathbf{1 Z})$. It supports the experimental result of preparation of the exciplex 1Z·2b* (Ex1: B), and nothing of photoadduct via a biradical (D), which is similar to biradical D in Figure 5, caused by the high barrier (C). The HOF of Ex1 is $34 \mathrm{kcal} / \mathrm{mol}$ lower than the sum of $\left(\mathbf{1 Z}+\mathbf{2} \mathbf{b}^{*}\right)$.

The clear graphs of Ex1 in Figure 3 and also "saddle" in Figure 6 are shown in Figure 4. The olefin part of $\mathbf{1 Z}$ in Ex1 shows small twist $\left(\Phi_{8123}=-19.8^{\circ}\right)$ and faces the benzene part of $\mathbf{2} \mathbf{b}$. The distances of 1(1z)-1(2b), and 2(1z)-2(2b) are $3.12 \AA$ and $2.82 \AA$ like as exciplexes of singlet [2+2]photoadditions [9, 14]. Four ester carboxyl groups of $\mathbf{2 b}$ all turn to olefin or allyl hydrogens, and the distances are 2.56, 2.66, 2.69 and $2.72 \AA$.

The stabilization of Ex1 is inferred from the $\pi / \pi^{*}$ interaction, four hydrogen bondings and $\mathrm{C}-\mathrm{H} / \pi$ interactions between the carbonyl oxygens or $\pi$ components, and $\mathrm{C}(\pi)$-H or allylic hydrogens observed in B conformation (Ex1). The dihedral angle $\Phi_{8123}(\mathbf{1 Z})$ in B was twisted $\left(20^{\circ}\right)$. We then checked two possibilities of : (a) ground state isomerization of equation 2 ,

$$
1 Z \cdot 2 b^{*} \rightarrow 1 E \cdot 2 b^{*}
$$

and (b) energy transfer of equation 3,

$$
1 \mathrm{Z} \cdot 2 \mathrm{~b}^{*} \rightarrow 1 \mathrm{E}^{*} \cdot 2 \mathrm{~b}
$$

For (a) the change to $94^{\circ}$ of the dihedral angle $\Phi_{8123}$ $\left(\mathbf{1 Z )}\right.$ in $\mathbf{1 Z} \cdot \mathbf{2} \mathbf{b}^{*}$ similar to Figure 2 needed high energy, $49 \mathrm{kcal} / \mathrm{mol}$, which shows the (a) process is impossible. We next calculated the energy for (b). 


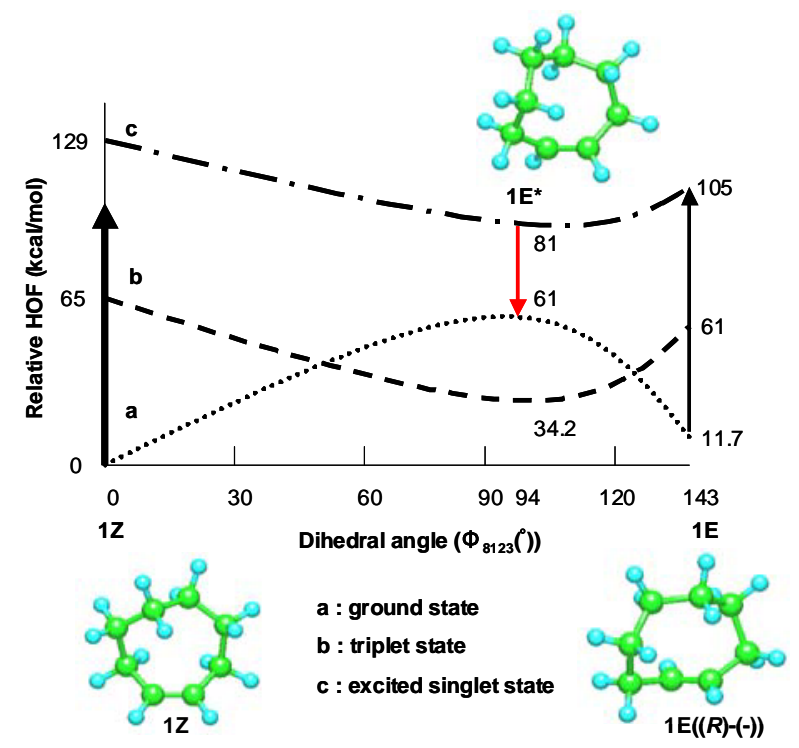

Figure 2. Relative energy (HOF) versus dihedral angle of $1 Z$ and $1 E$.

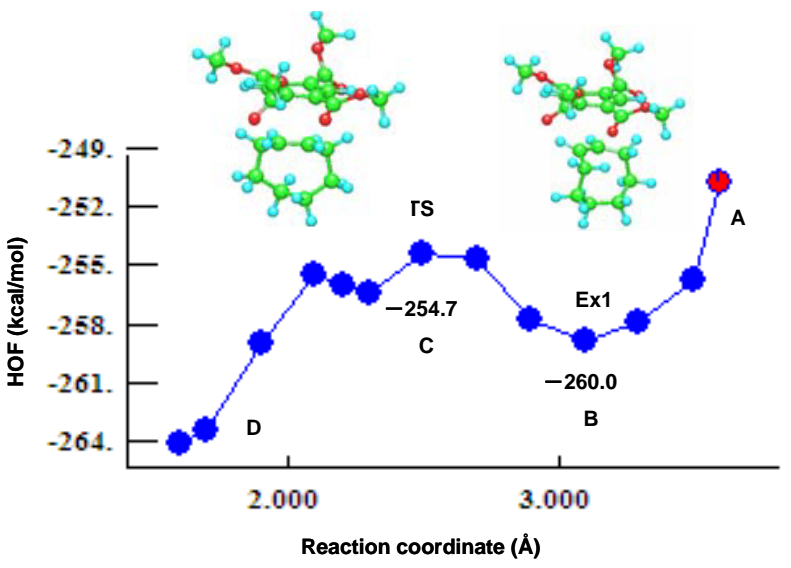

Figure 3. Interaction between $\mathbf{1 Z}$ and $\mathbf{2} \mathbf{b}^{*}$.

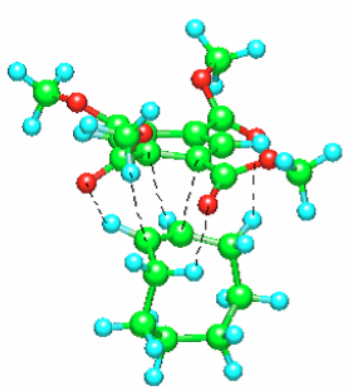

Ex1 structure

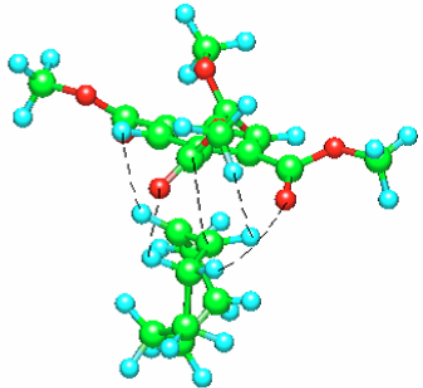

Saddle structure
Figure 4. Clear graphs of Ex1 and Saddle intermediates from $(\mathbf{1 z + 2 b})$ reaction in Figure 3 and Figure 6.

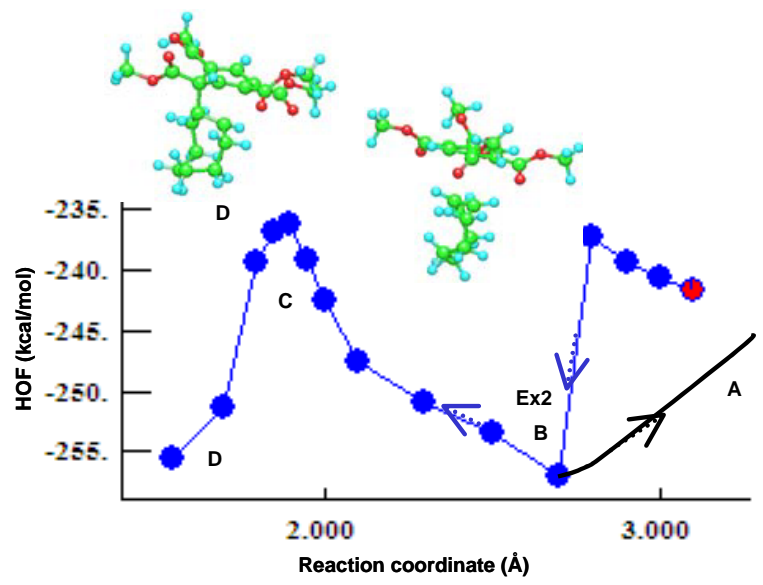

Figure 5. Interaction between $\mathbf{1 E}^{*}$ and $\mathbf{2 b}$.

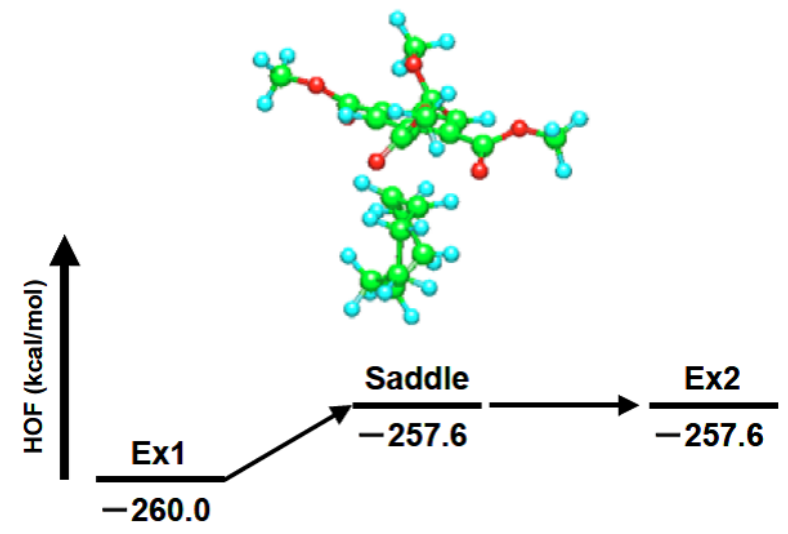

Figure 6. Energy transfer from $\mathbf{1 Z} \cdot \mathbf{2 b}^{*}(\mathbf{E x 1 )}$ to $1 E^{*} \cdot 2 b(\operatorname{Ex} 2)$.

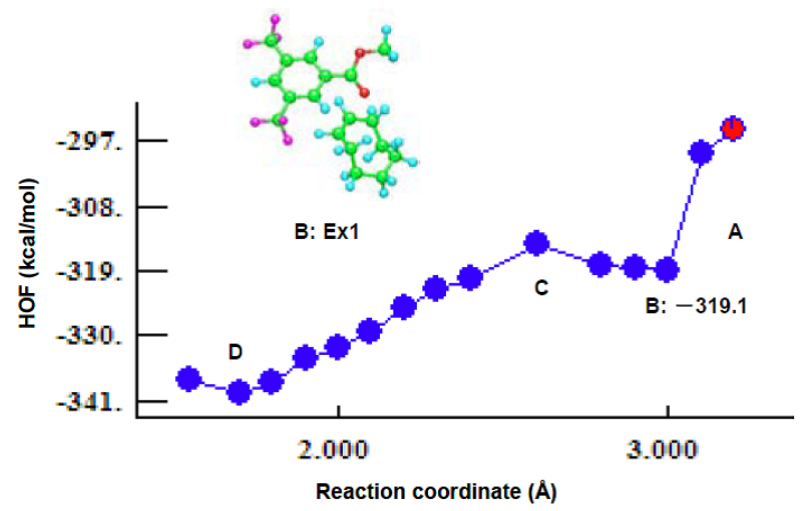

Figure 7. Interaction between $\mathbf{1 Z}$ and $\mathbf{2} \mathbf{c}^{*}$. 


\subsubsection{Exciplex Ex2 formation between $1 E^{*}$ and $2 b$, and energy transfer}

Figure 5 shows the interaction between $\mathbf{1 E}^{*}$ and $\mathbf{2 b}$ for Ex2 formation in equation (3). The Ex2 energy ( -257 $\mathrm{kcal} / \mathrm{mol}$ ) is $15 \mathrm{kcal} / \mathrm{mol}$ lower than the total energy of $\mathbf{1 E}^{*}$ and $\mathbf{2 b}$, and $3 \mathrm{kcal} / \mathrm{mol}$ higher than Ex1's in Figure 3. Ex2 is followed by quenching to $\mathbf{1 E}$ (and $\mathbf{1 Z}$ ). These data may be used for explanation of $\mathbf{E} / \mathbf{Z}$ ratio $\fallingdotseq 0.1$ at the $(\mathbf{1 Z + 2 b})$ photoreaction.

We also simulated the transition process from Ex1 $\left(\mathbf{1 Z} \cdot \mathbf{2} \mathbf{b}^{*}\right)$ to $\mathbf{E x 2}\left(\mathbf{1 E}^{*} \cdot \mathbf{2 b}\right)$ by use of the PM5 keyword, SADDLE, and show the data in Figure 6. The Saddle structure in Figure 6 has following data:

$$
\begin{aligned}
& \text { 1E: } \Phi_{8123}=-121.8^{\circ}, \Phi_{4567}=-59.2^{\circ}, \\
& \begin{aligned}
r(1(\mathbf{1 E})-1(\mathbf{2 b})) & =2.82 \AA, r(2(\mathbf{1 E})-2(\mathbf{2 b}))=3.30 \AA, \\
r(2(\mathbf{1 E})-5(\mathbf{2 b})) & =2.94 \AA, \mathrm{r}(\mathrm{CH}-\mathrm{O}=\mathrm{C}) \\
& =2.50,2.69,2.75,2.89(\AA),
\end{aligned} \\
& \mathrm{r}(1-\mathrm{H}(\mathbf{1 E})-1(\mathbf{2 b}))=2.39 \AA, \mathrm{r}(2-\mathrm{H}(\mathbf{1 E})-4(\mathbf{2 b}))=2.46 \AA .
\end{aligned}
$$

The data are near to those of $\mathbf{E x 1}$ except $\Phi_{8123}$, and like as a singlet exciplex of [3+2] addition type. Since the TS energy is very low, the energy transfer equilibrium is inferred to be smooth, and to be caused from many hydrogen bondings, and $\mathrm{CH} / \pi$ interactions (the distances: $2.3-2.9 \AA$ ) at the TS state.

\subsection{Sensitized photoisomerization of $1 \mathrm{Z}$ to 1E by 2c}

The $\mathbf{1 E} / \mathbf{1 Z}$ ratio of the sensitized reaction of $\mathbf{1 Z}$ to $\mathbf{1 E}$ by $\mathbf{2 c}$ is relatively high $(\mathbf{1 E} / \mathbf{1 Z}=0.6)$ [3]. Figure 7 shows the calculated energy diagram and stereochemistry by approach between $\mathrm{C} 2\left(\mathbf{2 c}^{*}\right)$ and $\mathrm{C} 1(\mathbf{1 Z})$. It suggests formation of the exciplex $\mathbf{1 Z} \cdot \mathbf{2} \mathbf{c}^{*}(\mathbf{E x 1})$ of the conformation $\mathrm{B}$ and HOF $(-319.1 \mathrm{kcal} / \mathrm{mol})$, which is $32 \mathrm{kcal} / \mathrm{mol}$ lower than the start $\left(\mathbf{1 Z}+\mathbf{2} \mathbf{c}^{*}\right)$. The stabilization is brought also from $\mathrm{H} / \mathrm{F}$ hydrogen bonding, placing alongside.

Figure 8 shows the interaction between $\mathbf{1 E}^{*}$ and $\mathbf{2 c}$ for $\operatorname{Ex} 2\left(\mathbf{1 E}^{*} \cdot \mathbf{2 c}:-319.9 \mathrm{kcal} / \mathrm{mol}\right)$. In $\operatorname{Ex} 1 \mathbf{1 Z}$ is on the $\mathbf{2} \mathbf{c}^{*}$, and in $\mathbf{E x} 2 \mathbf{1 E}^{*}$ is alongside of $\mathbf{2 c}$ possessing intermolecular interactions of hydrogen bonding, $\mathrm{CH} / \pi, \mathrm{H} / \mathrm{F}$ etc. The energy transfer process data $(-318.8 \mathrm{kcal} / \mathrm{mol}$ in Figure 9) from $\mathbf{E x 1}\left(\mathbf{1 Z} \cdot \mathbf{2} \mathbf{c}^{*}\right)$ to $\mathbf{E x 2}\left(\mathbf{1 E}^{*} \cdot \mathbf{2 c}\right)$ by use of keyword, SADDLE suggest that the isomerization is very smooth. $\mathbf{1 E} / \mathbf{1 Z}$ ratio by $\mathbf{2 c}$ is larger than that by $\mathbf{2 b}$ [3]. From difference between Figure 9 and Figure 6, Ex1> $\mathbf{E x 2}$ in the energy (HOF) is inferred to bring high $\mathbf{1 E / 1 Z}$ ratio.

\subsection{Chiral isomerization of $1 \mathrm{Z}$ to $1 \mathrm{E}$ by chiral menthyl 3,5-bis(trifluoromethyl) benzoate (2d)}

\subsubsection{Isomerization by $(R)$-menthyl isomer ( $2 \mathrm{dr})$}

Approach between excited singlet state $\left(\mathbf{2} \mathbf{d r} \mathbf{r}^{*}\right)$ of $\mathbf{2 d r}$ and $1 Z$ on PM5 program showed the existence of the exciplex 1Z·2dr" ${ }^{*}$ (Ex1 in Figure 10) $(\mathrm{HOF}=-366.7$ $\mathrm{kcal} / \mathrm{mol}$, dihedral angle $\left.\Phi_{8123}(\mathbf{1 Z})=-21.4^{\circ}\right)$ which is $16.9 \mathrm{kcal} / \mathrm{mol}$ lower than the total of $\left(\mathbf{1 Z}+\mathbf{2} \mathbf{d r} \mathbf{r}^{*}\right)$. Similar approach between the excited singlet state $\left(\mathbf{1 E}^{*}\right)$ of $\mathbf{1 E}$ and $\mathbf{2 d r}$ showed the existence of the exciplex $1 \mathbf{E}^{*} \cdot \mathbf{2 d r}$ $(\mathbf{E x 2}$ in Figure 10) $(\mathrm{HOF}=-368.9 \mathrm{kcal} / \mathrm{mol})$, which is $4.5 \mathrm{kcal} / \mathrm{mol}$ lower than the total of $\left(\mathbf{1 E}^{*}+\mathbf{2} \mathbf{d r}\right)$, and $2.1 \mathrm{kcal} / \mathrm{mol}$ lower than Ex1. The transition simulation from $\operatorname{Ex1}\left(\mathbf{1 Z} \cdot \mathbf{2 d r}{ }^{*}\right)$ to $\mathbf{E x 2}\left(\mathbf{1 E}^{*} \cdot \mathbf{2 d r}\right)$ by the keyword (SADDLE) showed the TS energy $(-366.6 \mathrm{kcal} / \mathrm{mol})$ and the steric conformation in Figure 10. Since the TS energy is very small $(0.1 \mathrm{kcal} / \mathrm{mol})$, the energy transfer leading to the isomer $\mathbf{1 E}$ is inferred to be very easy similarly to the case of 1c. The dihedral angle $\Phi_{8123}(\mathbf{1 Z})$ also changed from $-21.4^{\circ}$ to $-34.2^{\circ}$ and $-85.2^{\circ}$ in the transfer process, which suggests to go to $(R)-\mathbf{1 E}\left(\Phi_{8123}=-143^{\circ}\right)$ by oneside rotation.

3.4.2 Isomerization by $(S)$-menthyl isomer (2ds) and enantiodifferentiation

Similar approach between the unstable excited singlet state 2ds* and $\mathbf{1 Z}$ showed existence of the exciplex $\mathbf{1 Z} \cdot \mathbf{2 d s}{ }^{*}(\mathbf{E x 1}$ in Figure 11$)(\mathrm{HOF}=-366.3 \mathrm{kcal} / \mathrm{mol}$, $\left.\Phi_{8123}=-29.3^{\circ}\right)$, which is $0.4 \mathrm{kcal} / \mathrm{mol}$ unstable comparing with 1Z·2dr ${ }^{*}$ in 3.4.1. Exciplex 1E* 2ds $^{*}$ Ex2 in Figure 11$)(\mathrm{HOF}=-368.8 \mathrm{kcal} / \mathrm{mol})$ between $\mathbf{1 E}^{*}$ and $\mathbf{2 d s}$ is $2.5 \mathrm{kcal} / \mathrm{mol}$ lower than Ex1.

The transition simulation from Ex1 to Ex2 in Figure 11 showed low TS energy $(-366.5 \mathrm{kcal} / \mathrm{mol})$ almost the same as Ex1. By comparison between Figure 10 and Figure $11, \mathbf{1 Z}\left(\Phi_{8123}=-1.5^{\circ}\right)$ is inferred to be introduced more effectively to Ex1 and Ex2 by $\mathbf{2 d r}$ than 2ds, and to be followed by $(R)-\mathbf{1 E}\left(\Phi_{8123}=-143^{\circ}\right)$, which was examined experimentally by Inoue et al. [3]. The enantiodifferentiation may come from the proper intermolecular interactions of hydrogen bondings between $\pi$-H or allyl groups in $\mathbf{1 Z}$, and asymmetric ester carbonyl in $\mathbf{2 d r}$. The $\mathrm{CF}_{3}$ groups in $\mathbf{2 d r}$ are also thought to be effective for the interactions in the stereochemistry. 


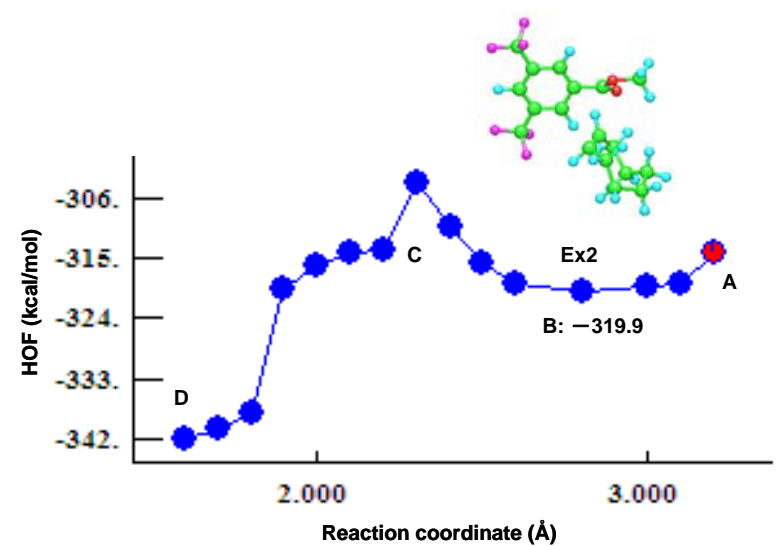

Figure 8. Interaction between $\mathbf{1 E}^{*}$ and $\mathbf{2 c}$.

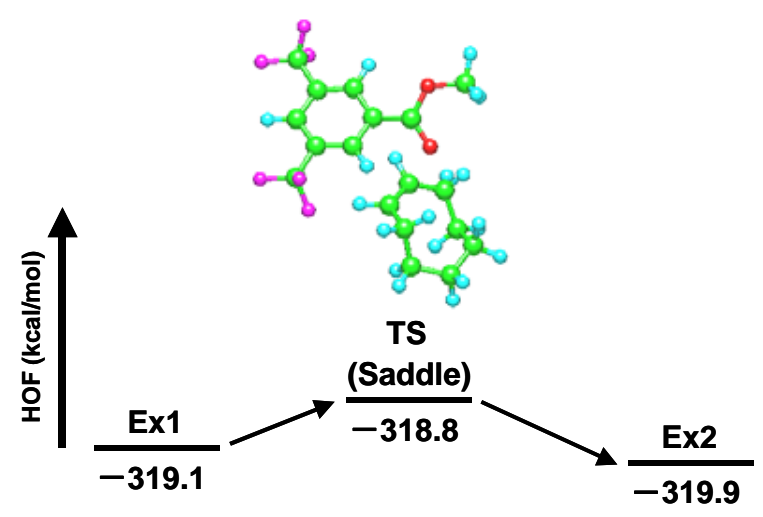

Figure 9. Energy transfer from $\mathbf{1 Z} \cdot \mathbf{2} \mathbf{c}^{*}(\mathbf{E x 1})$ to $\mathbf{1 E}^{*} \cdot \mathbf{2 c}$ (Ex2).

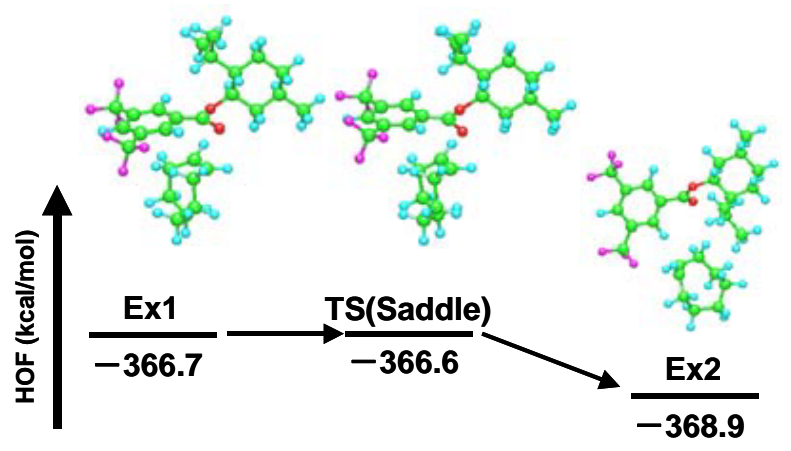

Figure 10. Ex1 and Ex2 from reaction $(\mathbf{1 Z}+\mathbf{2 d r})$ and the energy transfer.

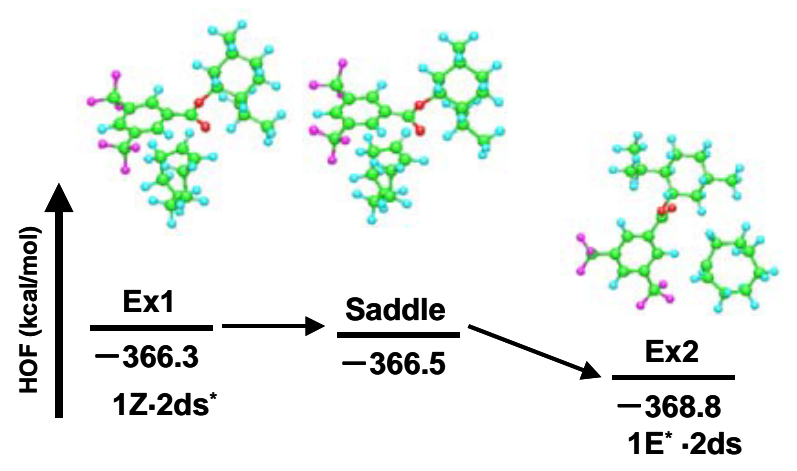

Figure 11. Ex1 and $\mathbf{E x} 2$ from reaction $(\mathbf{1 Z}+\mathbf{2 d s})$ and the energy transfer.
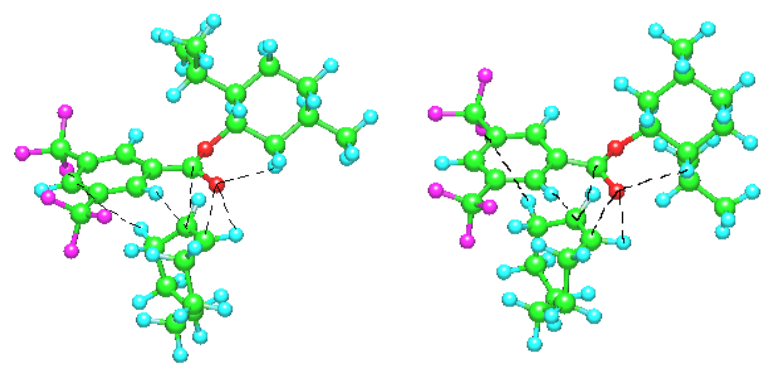

Saddle $/(1 Z+2 d r)$

Saddle $/(1 \mathrm{Z}+2 \mathrm{ds})$

Figure 12. Clear graphs of Saddle from 1Z·2dr ${ }^{*}$ (Figure 10) and Saddle from 1Z·2ds* (Figure 11).

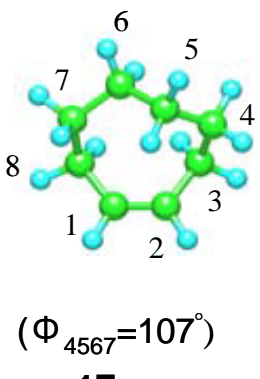

1Zc

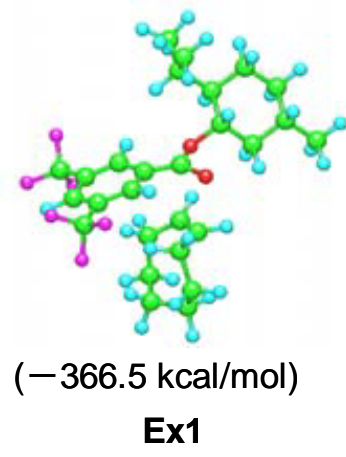

Figure 13. 1Zc and the $\mathbf{E x 1}\left(\mathbf{1 Z c} \cdot \mathbf{2 d s} \mathbf{s}^{*}\right)$ from reaction $(1 \mathrm{Zc}+\mathbf{2 d s})$. 
Figure 12 shows the clear graphs of Saddle in Figure 10 and of Saddle in Figure 11. The structural data of the former and the latter are as follows. Saddle/(1Z + 2dr): $\Phi_{8123}=-33.4^{\circ}, \Phi_{4567}=-109.8^{\circ}, \mathrm{r}(1(\mathbf{1 Z})-1(\mathbf{2 d r}))$ $=3.32 \AA, \mathrm{r}(1(\mathbf{1 Z})-2(\mathbf{C}=\mathrm{O}))=3.02 \AA, \mathrm{r}(2(\mathbf{1 Z})-2(\mathbf{O}=\mathrm{C}))$ $=2.63 \AA, \mathrm{r}(\mathrm{CH}-\mathrm{O}=\mathrm{C})=2.34,2.46(\AA), \mathrm{r}(\mathrm{CH} / \mathrm{F})=3.06$ $\AA, 1 \mathrm{Z} /$ iso-propyl (2dr): exo.

Saddle $/(\mathbf{1 Z}+\mathbf{2 d s}): \Phi_{8123}=-28.2^{\circ}, \Phi_{4567}=-109.8^{\circ}$, $\mathrm{r}(1(\mathbf{1 Z})-1(\mathbf{2 d s}))=3.32 \AA, \mathrm{r}(1(\mathbf{1 Z})-2(\mathrm{C}=\mathrm{O}))=3.07 \AA$, $\mathrm{r}(2(\mathbf{1 Z})-2(\mathrm{O}=\mathrm{C}))=2.71 \AA, \mathrm{r}(\mathrm{CH}-\mathrm{O}=\mathrm{C})=2.45,2.57(\AA)$, $\mathrm{r}(\mathrm{CH} / \mathrm{F})=2.99 \AA, \mathbf{1 Z} /$ iso-propyl (2ds): endo.

The saddle/ $(\mathbf{1 Z}+\mathbf{2 d r})$ seems to have advantages in $\pi / \pi^{*}$, and hydrogen bonding interactions, ionic interactions by ester carbonyl, and steric and conformational factors by the iso-propyl group. The smaller $\mathrm{r}(\mathrm{CH} / \mathrm{F})$ in the latter saddle also suggests delicate balance between their interactions and steric factors. They are inferred to bring smaller advantage in $\Phi_{8123}$ of the former, which is followed by $(R)-\mathbf{1 E}$.

$\mathbf{1 Z}\left(\Phi_{4567}=-107^{\circ}\right)$ has a conformational isomer 1Zc of opposite $\Phi$ signs. The isomer $1 \mathbf{Z c}$ was simulated to become rich $(S)-\mathbf{1 E}$ or not by use of $\mathbf{2 d s}$ as shown in Figure 13. Thus the energy Ex1 (1Zc·2ds $\left.{ }^{*}\right)(-366.5 \mathrm{kcal} / \mathrm{mol})$ was lower than that of the diastereomeric exciplex Ex1 $\left(\mathbf{1 Z c} \cdot 2 \mathbf{d r}^{*}\right)(-364.4 \mathrm{kcal} / \mathrm{mol})$. The latter data also suggests some margin of error [10,17]. By comparison of three steric structures of Ex1 in Figures 10, 12, 13, we infer that isopropyl group in the menthyl substituent is more of hindrance than an interaction.

Pirrung et al.[20] recently demonstrated that $(R)$ riched $(E)$-cyclooctene $(\mathbf{1 E})$ was more effective than the $(S)$-riched 1E as ethylene receptor (ETR1) antagonist. Since ethylene is used as a hormone to control physiological processes of plants, the enantiomeric selectivity may give a search for the asymmetric ETR 1 protein-composed environment. The enantiomerically enriched $\mathbf{1 E}$ was prepared by the method of photosensitized isomerization of $\mathbf{1 Z}$ using $(R)$ - and $(S)$-hexakis (1-methylheptyl) benzenehexacarboxylates as effective chiral sensitizers, which were developed by Inoue et al. [3].

\section{Conclusion}

Interesting experimental results on enantiodifferentiating photoisomerization of $(Z)$-cyclooctene $(\mathbf{1 Z})$ to chiral $(E)$ isomer (1E) sensitized by chiral polyalkyl benzenepolycarboxylates (2a-2d) were elucidated on the energy and stereochemical profile by molecular simulation by use of MOPAC-PM5 program.

Energy, stereochemistry, and equilibrium of ground states of $\mathbf{1 Z}$ (and conformer 1Zc), 1E, 2(2b-2d) and the excited singlet states of $\mathbf{1} \mathbf{Z}^{*}, \mathbf{1} \mathbf{E}^{*}, \mathbf{2}^{*}\left(\mathbf{2} \mathbf{b}^{*}-\mathbf{2} \mathbf{d}^{*}\right)$ were first elucidated. One asymmetric conformar $\mathbf{1 Z}\left(\Phi_{4567}=\right.$ $-107^{\circ}$ ) was inferred to have the preferential one-side rotation to go to $(R)-\mathbf{1 E}\left(\Phi_{8123}=-143^{\circ}\right)$ by photoisomeriza- tion. The photoisomerizations between $\mathbf{1 Z}$ and $\mathbf{2}$ were inferred to proceed via two exciplexes $\mathbf{E x 1}\left(\mathbf{1 Z} \cdot \mathbf{2}^{*}\right)$ and $\mathbf{E x} 2$ $\left(\mathbf{1 E} \mathbf{E}^{*} \cdot \mathbf{2}\right)$. The transition state (TS) for the energy transfer process is low and it is followed by quenching to $\mathbf{1 E}$.

The sensitization ratio $\mathbf{1 E} / \mathbf{1 Z}$ may be related to the energy difference ( $\triangle \mathrm{HOF}$ ) between each $\mathbf{E x 1}$ and $\mathbf{E x} \mathbf{2}$ as follows. Since $\mathbf{2 b}$ gives stable Ex1 because of $\pi^{*} / \pi$ and four ester-carbonyls for the effective intermolecular interactions such as $\mathrm{C}=\mathrm{O} / \mathrm{HC}$ ( $\pi$ and allyl) hydrogen bondings, the $\mathbf{1 E} / \mathbf{1 Z}$ ratio is low (0.1) [3]. As 2c gives stable Ex2 because of alongside interactions by the meta- $\mathrm{CF}_{3}$ and ester carbonyl for $\mathbf{E x} \mathbf{2}$, the $\mathbf{1 E} / \mathbf{1 Z}$ ratio is relatively high (0.6) [3].

The enantiodifferentiating photoisomerization of asymmetric $\mathbf{1 Z}$ to chiral $\mathbf{1 E}$ by chiral $\mathbf{2}$ may be estimated by calculation of diastereomeric Ex1 energy and TS energy for Ex2. Enantiomeric $(R)-\mathbf{1 E}$ via low Ex1 and small TS process from $\mathbf{1 Z}$ by $\mathbf{2 d r}$ (or $\mathbf{1 Z c}$ by $\mathbf{2 d s}$ ) was speculated to be preferred. The preference depends on sum of the steric interaction and repulsion energies including hydrogen bondings between asymmetric $\mathbf{1 Z}$ and enantiomeric $\mathbf{2 d} \mathbf{d}^{*}$. It will be influenced by $\mathbf{2 d}$ substituents and environment.

Such various and weak interactions are caused from intra- and intermolecular interactions and the entropycontrol factors of the reaction temperature etc., may happen chirality control of the photosensitized isomerization in solution.

We hope that our molecular simulation by $\mathrm{MO}$ method for enantiodifferentiating phenomena by weak intra- and intermolecular interactions suggests some ideas for development of such interesting interaction environments like enzymes.

We are grateful to Prof. Y. Inoue at Osaka University for advice and encouragement to this simulation research. We also thank Ms. T. Ooto at Kagoshima University for paper work.

\section{References}

[1] Y. Inoue, V. Ramamurthy, Chiral Photochemistry, Marcel Dekker, New York (2004).

[2] Y. Inoue, T. Yokoyama, N. Yamasaki, A. Tai, Nature, 341, 225 (1989).

[3] Y. Inoue, N. Yamasaki, T. Yokoyama, A. Tai, J. Org. Chem., 57, 1332 (1992).

[4] Y. Inoue, E. Matsushima, T. Wada, J. Am. Chem. Soc., 120, 10687 (1998).

[5] T. Mori, Y. Inoue, CRC Handbook of Organic Photochemistry and Photobiology, Second edition, 
edit. by W. Horspool, E. Lenci, CRC Press (2003), Chap.16-1.

[6] I. Fleming, Frontier Orbitals and Organic Chemistry Reactions, John Wiley \& Sons, Ltd (1976).

[7] J. L. Broeker, E. Eksterowicz, A. Z. Belk, K. N. Houk, J. Am. Chem. Soc., 117, 1847 (1995).

[8] T. Suishu, T. Obata, T. Shimo, K. Somekawa, Nippon Kagaku Kaishi, 2000, 167.

[9] T. Shimo, K. Somekawa, CRC Handbook of Organic Photochemistry and Photobiology, Second edition, edit. by W. Horspool, E. Lenci, CRC Press (2003), Chap.82-1.

[10] Y. Odo, T. Shimo, K. Hori, K. Somekawa, Bull. Chem. Soc. Jpn., 77, 1209 (2004).

[11] H. I. Omar, T. Shimo, K. Somekawa, J. Mol. Struct.(Theochem), 763, 115 (2006).

[12] T. Shimo, K. Sato, W. Wang, T. Obata, T. Iwanaga, T. Shinmyozu, K. Somekawa, Bull. Chem. Soc. Jpn., 81, 894 (2008).
[13] J. J. P. Stewart, MOPAC2002 Manual, Fujitsu Limited (2001).

[14] D. Tokunaga, T. Shimo, H. Hashimoto, T. Ooto, K. Somekawa, J. Comput. Chem. Jpn., 6, 283 (2007).

[15] K. Hori, S. Yamasaki, Keisan-Kagaku-Jikken, Maruzen (1995), 43.

[16] J. J. P. Stewart, Computational Chemistry-MOPAC Home Page.

http://www.MOPAC2007.com

[17] S. Sakaki, Kagaku-Sousetsu, No.47, GakkaiShuppan Center (2000), 179.

[18] S. Tokita, K. Somekawa, Ryoshi-Kagaku no Kiso, Shokabo (2005), 147.

[19] H. Sakiyama, A. Kazama, S. Suzuki, Y. Nishida, J. Comput. Chem. Jpn., 7, 27 (2008).

[20] M. C. Pirrung, A. B. Bleecker, Y. Inoue, F. I. Roseifuwz, N. Sugawara, T. Wada, Y. Zou, B. M. Binder, Chemistry \& Biology, 15, 313 (2008). 